\title{
Ärztegesellschaft des Kantons Bern - heute und in Zukunft
}

Jürg Schlup

Präsident der Ärztegesellschaft des Kantons Bern

Korrespondenz:

Dr. med. Jürg Schlup exec. MBA HSG

Präsident Ärztegesellschaft des Kantons Bern Bolligenstrasse 52

CH-3006 Bern

schlup@hin.ch

\section{Heute - Strukturwandel getrieben von den Kosten}

Der Strukturwandel in Stichworten:

- Die Bevölkerung drängt nach Gesundheit.

- Die Patienten fordern empört ihr Recht auf Gesundheit ein und haben sich weit entfernt von der einstigen demütigen Dankbarkeit.

- Die Ärzteschaft leistet Dienst nach Tarif entschädigt und nicht länger von der Gesellschaft honoriert.

- Einige Ärzte fürchten zunehmend die Freiheit, weil sie Verantwortung bedeutet.

- Der Wandel ermöglicht Entwicklungen, die bislang unmöglich schienen.

Der rasche Strukturwandel hat dazu geführt, dass die Berner Ärztegesellschaft seit dem Jahr 2000 erstmals in ihrer Geschichte ärzteeigene Firmen gegründet hat drei an der Zahl:

- das erste arzteigene Trust Center der Schweiz, PonteNova AG; zwecks Organisation des Datenflusses zwischen Ärzteschaft und Krankenkassen, Aufbau eigener Datenbanken und Erstellung von Statistiken;

- eine Schule für Medizinische Praxisassistentinnen, MedA AG;

- ein Call Center, Medphone AG; zwecks Errichtung und Betrieb einer arzteigenen Notfallzentrale zur Erfüllung der mit der ärztlichen Berufsausübungsbewilligung verbundenen Notfalldienstpflicht.

Mit letztgenannter Firmengründung haben wir 2006 den Anna-Seiler-Preis gewonnen - für innovative Lösungen im Gesundheitswesen.

Seit 2005 ist die Ärztegesellschaft als Berufsverband Mitglied von Berner KMU, dem mit 22000 Mitgliedern grössten kantonalen Gewerbeverband der Schweiz. Sie ist dort zweitgrösster Berufsverband, damit wichtiges Mitglied und im Vorstand vertreten. Diese starke Partnerschaft ist angesichts des raschen Strukturwandels im Gesundheitswesen besonders wichtig. Plattformen, die der Ärztegesellschaft durch diese Partnerschaft offenstehen, werden für Abstimmungskämpfe, parlamentarische Vorstösse und Vernehmlassungen genutzt. Berner KMU ist ein gewichtiger Türöffner für politische Gremien und unterstützte die Ärztegesellschaft bislang u.a. in den Bereichen Chefarzthonorare und Institut für Hausarztmedizin.

Seit 2006 ist die Ärztegesellschaft Mitglied der Organisation der Arbeitswelt OdA Gesundheit BE; der kantonalen Arbeitgeberorganisation im Gesundheitswesen und dort Mitglied des Vorstandes.

2003 begann die Ärztegesellschaft kantonsweit den ambulanten ärztlichen Notfalldienst zu reorganisieren. Ein Call Center wurde aufgebaut, Statuten und Reglemente revidiert, zwei umsetzungsorientierte Klausurtagungen mit den Unterorganisationen durchgeführt und eine finanzielle Beteiligung des Kantons an den Organisationskosten des ambulanten ärztlichen Notfalldienstes zu erreichen versucht. Letzteres gelang 2007. Heute bestehen vertragliche Zusammenarbeitsformen zwischen ärztlichen Notfallkreisen und zehn Spitälern öffentlicher und privater Trägerschaften kantonsweit. Dieser Prozess ist nicht abgeschlossen.

Innert vier Jahren, 2004-2008, hat die Ärztegesellschaft drei TARMED-Anschlussverträge verhandelt und abgeschlossen, zweimal auf Kündigungsdruck der Kassen. Damit sank die Halbwertzeit von Kassenverträgen mit der praktizierenden Ärzteschaft in unserm Kanton

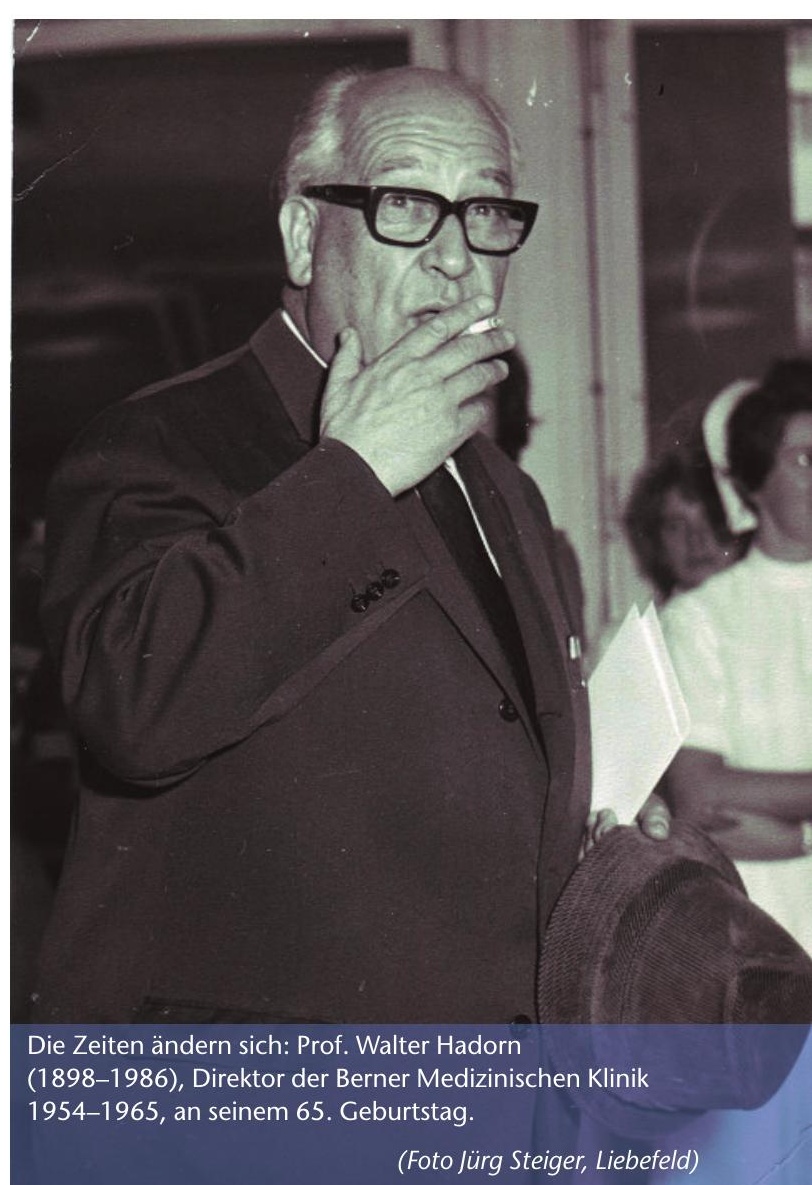




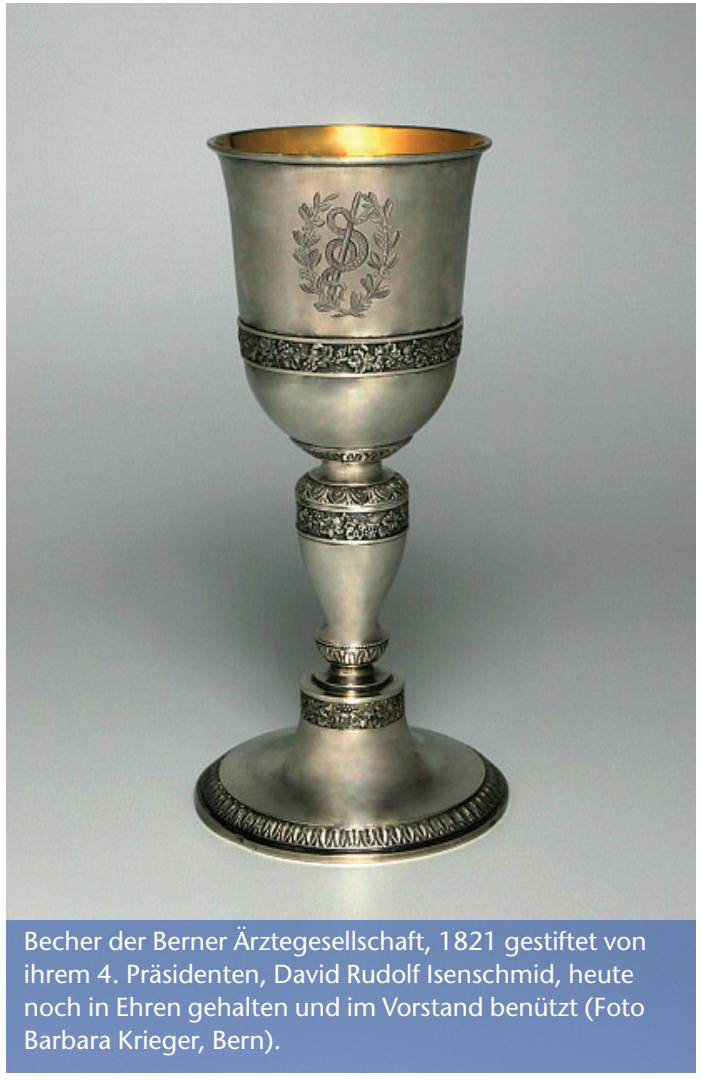

auf ein Jahr bzw. praktisch auf den Papierwert. Dies als Hinweis auf die aktuelle Vertragskultur im Gesundheitswesen nicht allein im Kanton Bern.

Zur Förderung des elektronischen Datenaustauschs zwischen Spital und Praxis setzt die Ärztegesellschaft seit sechs Jahren einen Delegierten ein, der sich für den integrierten Einsatz von Informations- und Kommunikationstechnologie zur Gestaltung, Unterstützung und Vernetzung aller Prozesse und Teilnehmer im Gesundheitswesens des Kantons einsetzt. Momentan erfüllen 10\% der Ärztinnen und Ärzte die geforderten technischen Voraussetzungen für integriertes E-Health.

Seit 2008 arbeitet die Ärztegesellschaft an einem Projekt zur Sicherstellung der ärztlichen Versorgung einer Randregion. Neben dem regionalen Bezirksverein konnten das regionale Spitalzentrum, regionale Arbeitgeber, die Ärztekasse und die lokalen Gemeinden für dieses Projekt gewonnen werden.

Die Berner Ärztegesellschaft zählt heute 3000 Mitglieder und 32 Unterorganisationen. Damit ist sie die zweitgrösste kantonale Ärztegesellschaft der Schweiz. Sie engagiert sich für akzeptable kantonale Rahmenbedingungen für die Ärzteschaft; für eine qualitativ hochstehende, regionale ärztliche Versorgung der Pa- tientinnen und Patienten sowie für Lösungen im Gesundheitswesen, die zukunftsgerichtet sind, partnerschaftlich erarbeitet werden und die Würde und Autonomie der betroffenen Menschen respektieren. Die Ärztegesellschaft pflegt dazu regelmässige Aussenkontakte zur Kantonsregierung, santésuisse, Versicherern, Parteien und nichtärztlichen Berufsverbänden.

\section{In Zukunft - Strukturwandel getrieben vom Fachkräftemangel}

Früher war schon die Zukunft viel schöner, meinte Karl Valentin - «Ärzte haben einen der schwierigsten Berufe überhaupt» [1]. «Brillante junge Studenten und Ärzte wenden sich von der Medizin ab; sie wollen nicht mit Schuldgefühlen belastet sein, wenn sie doch etwas Sinnvolles tun» [2].

Für eine grosse regionale Ärztegesellschaft wie diejenige des Kantons Bern ist die Zukunft als Mitspieler im Gesundheitswesen viel versprechend und arbeitsintensiv. Die Gründe im Folgenden.

Wir erleben das Wiedererstarken von Werten; dazu ein Beispiel: Absolventinnen einer Eliteuniversität möchten lieber Kinder als Karriere und Geld [3]. Dieser Wertewandel erfasst auch das Gesundheitswesen: Kosten sind nicht alles, Qualität wird wichtiger werden, so auch Transparenz und Vertrauen. In diesem Wandel werden neue Lösungsansätze - auch von regionalen Ärzteorganisationen - gefragt sein.

Weil man den Kostenanstieg nicht stoppen, allenfalls leicht bremsen kann [4], wird Zusammenarbeit noch wichtiger werden. Da auf der Ebene des Bundes eine nationale, kohärente Gesundheitspolitik weiterhin fehlt [5], wird diese Zusammenarbeit regional und überregional Raum greifen. Um gemeinsam nach Lösungen zu suchen, werden runde Tische mit allen Akteuren des Gesundheitswesens bald im Trend liegen. Auf der kantonalen Ebene ist man dabei heute schon weiter als auf der nationalen. Regionale Berufsverbände wie die Ärztegesellschaft sind heute an regionalen runden Tischen gefragt.

Unser Gesundheitswesen wird besser vernetzt sein. Die Einführung von DRG wird die integrierte Versorgung beflügeln. Eine grosse regionale Ärztegesellschaft wie jene Berns kann dabei eine hilfreiche Rolle spielen. Sie kann mit ihrem bereichsübergreifenden regionalen Netzwerk und demjenigen ihrer Mitglieder Lösungen ermöglichen für eine integrierte regionale Gesundheitsversorgung von Prävention über Spitex, Praxis, Konsiliardiensten, Ambulatorien, Spitälern, Unikliniken, Rehabilitationsangeboten und Behörden. Sie kann den Strukturwandel in der ambulanten ärztlichen Versorgung unterstützen, mit neuen Strukturen und neuen Arbeitsmodellen.

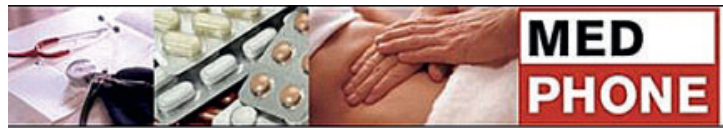


Die Wirtschaftskrise wird auf die Prämien durchschlagen (Börsenverluste der Versicherer) und das fehlende Wirtschaftswachstum die Finanzierung des Gesundheitswesens deutlich erschweren. Vorübergehend werden dadurch die staatlichen Regulierungsanstrengungen zunehmen. Regierungen brauchen dazu Ansprechpartner auf der regionalen Ebene. Eine grosse kantonale Berufsorganisation wie die Berner Ärztegesellschaft ist dafür geeignet und gesucht. Die volkswirtschaftlichen Kosten werden wichtiger werden, d.h. der kollektive Nutzen wird ein Thema. Damit wird die regionale Ressourcenbereitstellung [6] diskutiert werden und die Meinung regionaler Ärztegesellschaften wird gefragt sein. «Health care is on a collision course with patient needs and economic reality. To reform health care, we must reform the nature of competition itself. The locus of competition has to shift from Who pays? to Who provides the best value?» [7]. Dem Patientennutzen gehört die Zukunft und nicht den tiefen Kosten - meint damit die US-amerikanische Professorin Elisabeth Olmsted Teisberg.

Der Markt gewährleistet die Versorgungssicherheit nicht automatisch, und daher muss der Staat eine regulierende Funktion übernehmen, um der ganzen Bevölkerung den Zugang zur medizinischen Versorgung zu finanziell tragbaren Bedingungen zu garantieren [8]. Solange die Kantone diese überregionale Versorgungs- sicherheit garantieren, sind kantonale Ärztegesellschaften wie diejenige des Kantons Bern valable Partner im Gesundheitswesen.

\section{Literatur}

1 Kesseli B. «Ärzte haben einen der schwierigsten Berufe überhaupt.» Interview mit Gianfranco Domeninghetti, Gesundheitsökonom. Schweiz Ärztezeitung. 2007;88(31/32):1325-9.

2 Frey F. Rede am Dies academicus 2007. Universität Bern. S. 11.

3 Gelernter D. Die Computer-Nutzer sollten endlich einmal protestieren: Interview. GDI-Impuls. 2008; (1):102-8.

4 Ruey C. «Man kann den Kostenanstieg nicht stoppen». NZZ am Sonntag, 11. Januar 2009.

5 Imhasly P. Nationaler Gesundheitsbericht 2008. Schweizerisches Gesundheitsobservatorium OBSAN. Teil IV. Neuenburg: Bundesamt für Statistik; 2008.

6 Rationierung im Gesundheitswesen: Einschätzung und Empfehlungen. Projekt Zukunft Medizin Schweiz - Phase III. Schweizerische Akademie der medizinischen Wissenschaften SAMW: 2007.

7 Porter ME, Olmsted Teisberg E. Redefining Health Care: Creating Value-Based Competition on Results. Boston: Harvard Business School Press; 2006.

8 Domenighetti G, Crivelli L. Aufhebung des Vertragszwangs. Solothurn: santésuisse; 2001. 